04

\title{
Зондовый метод измерения коэффициента Бома, толщины зондового слоя объемного заряда и массы ионов
}

\author{
() П.Е. Машеров, В.А. Рябый \\ НИИ прикладной механики и электродинамики Московского авиационного института (национального \\ исследовательского университета), \\ 125080 Москва, Россия \\ e-mail: riaby2001@yahoo.com
}

(Поступило в Редакцию 3 октября 2016 г. В окончательной редакции 26 апреля 2018 г.)

\begin{abstract}
Предложено измерять коэффициент Бома, толщину зондового слоя объемного заряда и массу ионов в максвелловской плазме низкого давления на основе результатов стандартных зондовых измерений, используя эффект Бома, закон Больцмана и „закон 3/2“ в случае их справедливости для изучаемой плазмы. Реализация данной возможности была обеспечена аккуратной зондовой диагностикой высокочастотной ксеноновой плазмы индукционного разряда при давлении $2 \mathrm{mTorr}$. Анализ полученных результатов показал справедливость указанных законов и эффекта Бома с „инженерной“ точностью, что позволило найти коэффициент Бома $C_{\mathrm{BCyl}} \approx 1.23$ для цилиндрического зонда, составивший основу предложенного метода.
\end{abstract}

DOI: 10.21883/JTF.2018.12.46780.2051

\section{Введение}

Данное исследование основано на аккуратной зондовой диагностике индукционной ксеноновой плазмы при давлении $p=2 \cdot 10^{-3}$ Torr. В настоящее время большинство проблем этой диагностики разрешено вполне эффективно [1]. Например, зондовая станция Plasma Sensors VGPS Probe System, упомянутая в обзоре [1] и описанная на сайте [2], обеспечивает аккуратную диагностику любой плазмы, включая рабочие среды высокочастотных (ВЧ) разрядов. Анализ объективности этих измерений [3] и особенностей техники обработки вольт-амперных характеристик (BAX) зондов показал, что данная станция позволяет измерять температуру $T_{e}$ и концентрацию $n_{e}$ электронов с погрешностью порядка $\pm 10 \%$ и с гораздо меньшей ошибкой - плазменные потенциалы $V_{f}$ (плавающий) и $V_{s}$ (потенциал пространства) с соответствующей последнему плотностью электронного тока насыщения $j_{e s}$. Эта ситуация стимулировала поиски новых возможностей зондовой диагностики плазмы. Настоящая работа посвящена описанию зондового метода измерений толщины $\delta$ зондового слоя объемного заряда и массы ионов $M_{i}$ на основе эффекта Бома, закона Больцмана и ,закона 3/2“для цилиндрических зондов.

\section{Сущность рассматриваемой задачи}

В изотропной плазме с максвелловской функцией распределения электронов по энергиям (EEDF) справедливы эффект Бома, закон Больцмана для бесстолкновительного движения электронов в тормозящем потенциальном электрическом поле и ,закон 3/2“ в форме уравнений Чайлда-Ленгмюра-Богуславского (CLB). Эти особенности, часто встречающиеся в газоразрядной плазме низкого давления, позволили расширить возможности зондовой диагностики плазмы.

Согласно эффекту Бома [4], между зондовым слоем объемного заряда и невозмущенной плазмой формируется квазинейтральный переходный слой, в котором ионы ускоряются до электронных скоростей на границе зондового слоя $v_{e}=\left(2 k T_{e} / M_{i}\right)^{1 / 2}$, где $k-$ постоянная Больцмана. Они приходят к его внешней поверхности с плотностью тока, которая в случае цилиндрического зонда под плавающим потенциалом представлена в [4] как

$$
j_{i f l}=0.4 e n_{e}\left(2 k T_{e} / M_{i}\right)^{1 / 2}=0.4 e n_{e}\left(2 e T_{e} / M_{i}\right)^{1 / 2} .
$$

Здесь $e-$ элементарный заряд, $T_{e}-$ в первом варианте (1) выражена в $\mathrm{K}$, во втором - в $\mathrm{eV}$, а $0.4=C_{\mathrm{BCyl}}-$ определенный в [4] коэффициент Больцмана для цилиндрического зонда. Такая геометрия зондов наиболее популярна среди экспериментаторов из-за их конструктивной простоты, поэтому им и посвящена настоящая работа.

Данный эффект интересен функциональной связью плотности ионного тока $j_{i f l}$ на зонд под плавающим потенциалом с массой ионов $M_{i}$, что создает принципиальную возможность анализа массового состава ионной компоненты плазмы.

Плотность ионного тока насыщения на собирающую поверхность плавающего зонда $j_{i f}$ может быть найдена через плотность электронного тока насыщения $j_{e s}$, подчиняющуюся в максвелловской плазме закону Больцмана $n_{e f}=n_{e 0} \exp \left(-\Delta V_{f} / T_{e}\right)$, где $n_{e f}-$ концентрация электронов около собирающей поверхности зонда под плавающим потенциалом, $n_{e 0}-$ концентрация электронов на внешней границе зондового слоя, $\Delta V_{f}=V_{s}-V_{f}-$ разность между потенциалом пространства $V_{s}$ и плавающим потенциалом зонда $V_{f}$ 
при записи $T_{e}$ в $\mathrm{eV}$. Тогда на зонде под плавающим потенциалом из-за отсутствия тока в зондовой цепи

$$
j_{i f}=j_{e f}=j_{e s} \exp \left(-\Delta V_{f} / T_{e}\right) .
$$

Данный параметр, измеряемый цилиндрическим зондом Ленгмюра, может быть включен в классическую формулу Бома (1) с привлечением соотношения между собирающей поверхностью зонда и внешней поверхностью зондового слоя, равного отношению радиуса зонда $a$ к внешнему радиусу зондового слоя $R$ :

$$
j_{i f}(a / R)=j_{i f l}=C_{\mathrm{BCyl}} e n_{e}\left(2 e T_{e} / M_{i}\right)^{1 / 2} .
$$

Заметим, что разность этих радиусов $R-a$ определяет толщину $\delta$ зондового слоя объемного заряда, которая также представляет интерес для экспериментаторов. Обозначая $R / a=x$ и $x C_{\mathrm{BCyl}}=K_{\mathrm{BCyl}}-$ составному коэффициенту Бома, получаем вариант формулы Бома, содержащей измеряемый параметр $j_{i f}$ :

$$
j_{i f}=K_{\mathrm{BCyl}} e n_{e}\left(2 e T_{e} / M_{i}\right)^{1 / 2} .
$$

В этом выражении имеются три неизвестные величины - отношение радиусов $x=R / a$, подлежащий экспериментальному подтверждению коэффициент Бома $C_{\mathrm{BCyl}}$ и масса ионов $M_{i}$. Очевидно, что в случае

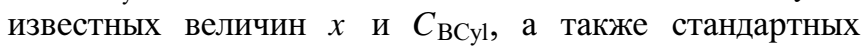
зондовых параметров плазмы $T_{e}, n_{e}$ и $j_{i f}$, формула (4) определяет среднюю массу ионов.

Для нахождения всех трех неизвестных нужны еще два независимых уравнения. Однако помимо формулы Бома имеется еще только одно соотношение для этих неизвестных - „закон 3/2“ [5], который в форме уравнения CLB для цилиндрического зонда под плавающим потенциалом записывается следующим образом:

$$
j_{i f}=\left(4 \varepsilon_{0} / 9\right)\left(2 e / M_{i}\right)^{1 / 2}\left(\Delta V_{f}^{3 / 2} / a^{2} x_{\mathrm{CLB}} A_{\mathrm{L}}\right),
$$

где $\varepsilon_{0}$ - диэлектрическая постоянная вакуума, а $A_{\mathrm{L}}-$ зависящий от $x_{\mathrm{CLB}}$ безразмерный параметр Ленгмюра [5]. Очевидно, что система из двух уравнений с тремя неизвестными не может быть разрешена однозначно. Предложенный в настоящей работе выход из положения заключается в разбиении исследования на два этапа, в каждом из которых система двух уравнений (4) и (5) будет содержать две неизвестные величины.

На первом этапе проводится специальный эксперимент с рабочим газом особой чистоты, подаваемым в безмасляный вакуум, т.е. при известной массе ионов $M_{i}$. Тогда система уравнений (4) и (5) позволяет провести оценки толщин зондового слоя $\delta_{\mathrm{CLB}}=R_{\mathrm{CLB}}-a=a\left(x_{\mathrm{CLB}}-1\right) \quad$ и коэффициента Бома $\left(C_{\text {BCyl }}\right)_{\text {CLB в }}$ вамках CLB-модели зондового слоя. Для этого в настоящей работе по результатам специального эксперимента в виде набора экспериментальных точек $\left(j_{i f}, n_{e}\right.$ и $\left.T_{e}\right)$ по формуле (4) были найдены величины составного коэффициента Бома $K_{\mathrm{BCyl}}=x C_{\mathrm{BCyl}}$.

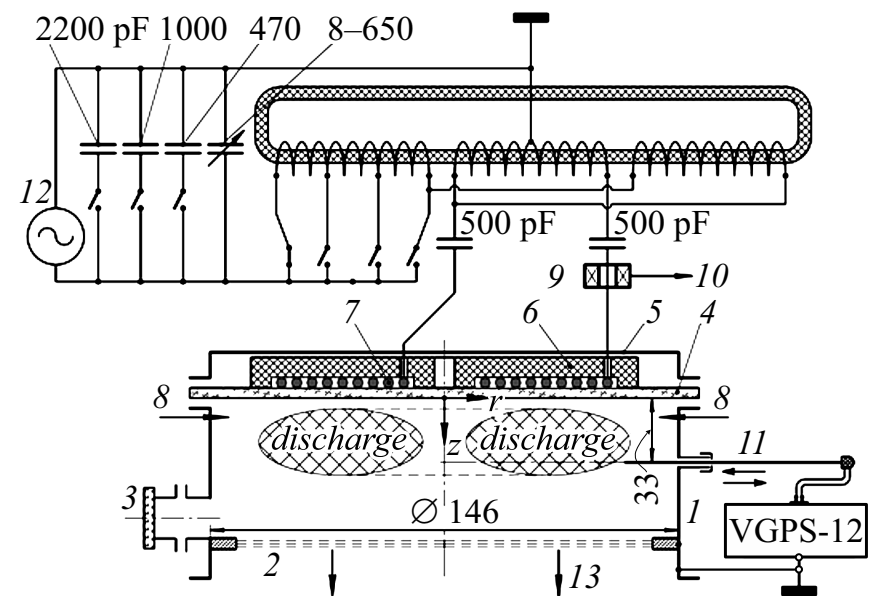

Рис. 1. Схемы ВЧИ установки и линии электропитания разряда: 1 - газоразрядная секция вакуумной камеры, 2 - ионнооптическая система, 3 - смотровое окно, 4 - разделительное кварцевое окно, 5 - крышка узла индуктора, $6-$ ферритовый сердечник, 7 - индуктор, 8 - подача ксенона, $9-$ пояс Роговского (Pearson mod. 2878), $10-$ к ВЧ-вольтметру, 11 - радиально подвижный цилиндрический зонд, 12 - ВЧ генератор.

Совместное решение уравнений (4) и (5) позволило найти соответствующие значения $x_{\mathrm{CLB}}$, определившие толщины зондовых слоев в рамках CLB-модели. Однако, как будет показано ниже, эта модель зондового слоя объемного заряда недостаточно четко обоснована физически, поэтому в нее будут внесены поправки, которые преобразуют оценки $x_{\mathrm{CLB}}$ в уточненные величины $x$, которые позволят найти соответствующий им коэффициент Бома $C_{\text {Всу1 }}$.

Вторым этапом решения системы уравнений (4) и (5) будет проведение эксперимента общего характера при наличии измеренного коэффициента Бома $C_{\mathrm{BCyl}}$ для нахождения толщин $\delta$ слоев объемного заряда на цилиндрическом зонде и средней массы ионов $M_{i}$.

Очевидно, что данный этап представляет собой предложенную в настоящей работе зондовую методику измерения $\delta$ и $M_{i}$, которая будет сформулирована после экспериментального уточнения коэффициента Бома $C_{\text {Всу1 в }}$ результате описанного ниже эксперимента с известной массой ионов ксенона $M_{i}=131 \mathrm{a.e.}=2.175 \cdot 10^{-25} \mathrm{~kg}$.

\section{Организация специального эксперимента с ВЧ индукционной плазмой}

Высокочастотный индукционный (ВЧИ) разряд в ксеноне возбуждался на частоте $2 \mathrm{MHz}$ в газоразрядной секции модели ВЧ ионного двигателя с плоским индуктором и ферритовым сердечником [6]. Схемы модели и линии ее электропитания представлены на рис. 1. 
Прототипом данной модели послужили патент [7] и его детальное исследование [8]. В проведенном специальном эксперименте ксенон особой чистоты $99.9999 \%$ подавался в вакуумную камеру объемом 1.91 с безмасляными средствами откачки. Негерметичность камеры выявлялась и устранялась с помощью японского течеискателя MSE-2000R. В результате предельный вакуум установки достиг $\sim 10^{-6}$ Torr. Расход ксенона составил $q=2 \mathrm{sccm}$, соответствующий весовому расходу $g \approx 0.2 \mathrm{~g} / \mathrm{s}$, что обеспечило динамическое разрежение в газоразрядной камере $p=2 \cdot 10^{-3}$ Torr.

Зондовая диагностика плазмы проводилась с помощью радиально подвижных прямых цилиндрических зондов Ленгмюра 11 (см. рис. 1), выполненных из вольфрамовой нити диаметром $0.15 \mathrm{~mm}$ с длиной измерительного отрезка $l_{p}=10 \mathrm{~mm}$. Зондовая нить размещалась в одном из каналов четырехканального керамического капилляра внешнего диаметра $0.88 \mathrm{~mm}$. Чертеж этого зонда представлен на рис. 2.

Керамический капилляр был помещен в заземленной стальной экранирующей трубке с внешним диаметром $1.6 \mathrm{~mm}$. Отрезок такой же трубки длиной $10 \mathrm{~mm}$ был зафиксирован около измерительного торца керамического капилляра и был электрически соединен со второй вольфрамовой проволочкой. Этот электрод с развитой собирающей поверхностью представлял собой опорный зонд, подключавшийся вместе с измерительным зондом к автоматизированной зондовой станции VGPS-12. Сигнал опорного зонда устранял значительную часть искажений зондовых ВАХ.

Особое внимание было уделено минимизации зондовых возмущений плазмы. Для этого были использованы зонды трех длин: 3,5 и $10 \mathrm{~mm}$ (см. рис. 2). Измерения показали [9], что с увеличением длины зондов их данные по $n_{e}$ вышли на насыщение, следовательно при длине зонда $l_{p}=10 \mathrm{~mm}$ влияние на $n_{e}$ рекомбинации заряженных частиц на зондодержателе оказалось пренебрежимо малым, т. е. рабочий зонд длины $l_{p}=10 \mathrm{~mm}$ контактировал с практически невозмущенной плазмой.

Управляющая программа станции VGPS-12 использует метод Дрювестейна, предусматривающий двойное дифференцирование зондовых ВАХ для регистрации EEDF, с которых начинается получение диагностических данных. Пример этих данных в форме функций

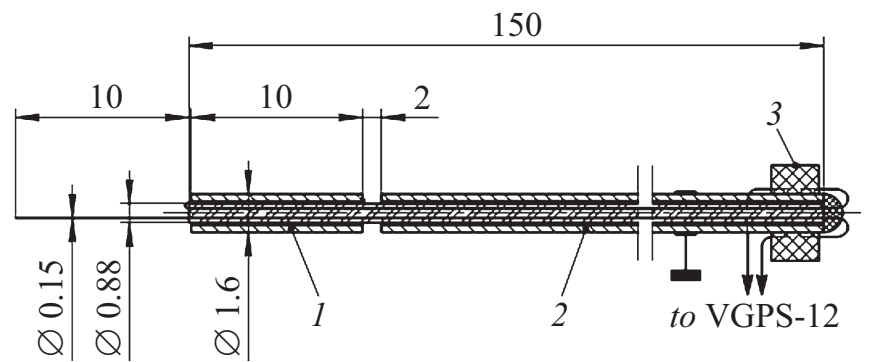

Рис. 2. Чертеж цилиндрического зонда: 1 - опорный зонд, 2 - защитный экран, 3 - узел разделки зондовых выводов.

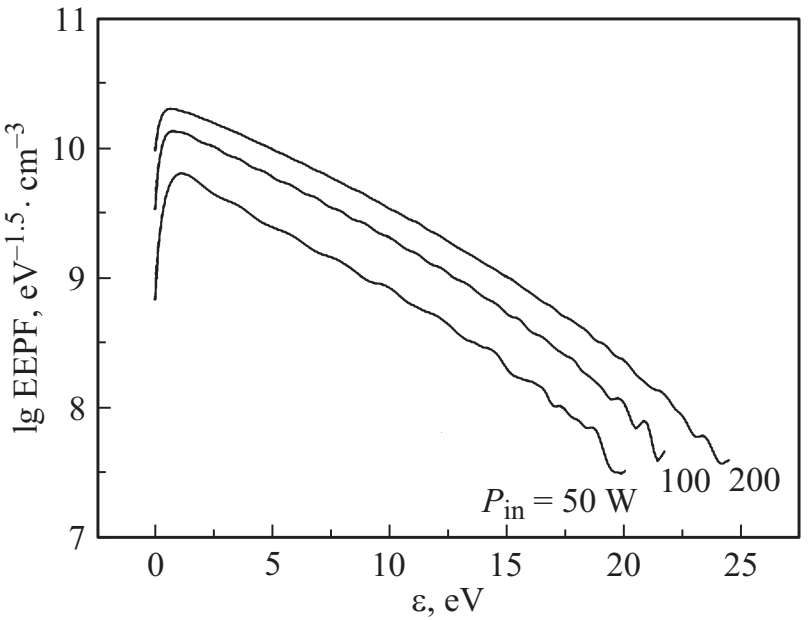

Рис. 3. Пример измеренных ЕEPF для $r=0$ и различных значений $P_{\text {in. }}$.

вероятности распределения электронов по энергиям $\mathrm{EEPF}=\mathrm{EEDF} / \varepsilon^{1 / 2}$ для осевой позиции зонда $(r=0)$ при падающей мощности ВЧГ $P_{\text {in }}=50,100$ и $200 \mathrm{~W}$ представлен на рис. 3.

Далее эти функции в рамках метода Дрювестейна интегрируются для получения $n_{e}$ и $T_{e}$. В максвелловской плазме полулогарифмические EEPF должны быть прямолинейными. В данном эксперименте такая особенность отсутствовала, но и отклонения измеренных EEPF от линейности были не слишком велики.

Оценка внешнего вида EЕРF представляет собой качественный анализ близости измеренных EEDF к максвелловской функции. Авторами предложен также и количественный вариант такого анализа в виде сравнения экспериментальных величин плотности электронного тока насыщения зонда $j_{e s}$ с расчетными значениями для изотропной максвелловской плазмы $j_{e s m}=(1 / 4) e n_{e}\left(8 k T_{e} / \pi m_{e}\right)^{1 / 2}$. Оказалось, что измеренные $j_{e s}$ были примерно на $20 \%$ ниже расчетных величин $j_{e s m}$. Этот факт был принят как „инженерная“ точность близости реальных EEDF к функции Максвелла.

При давлении $p=2 \mathrm{mTorr}$ все процессы взаимодействия электронов с цилиндрическим зондом диаметром $0.15 \mathrm{~mm}$ и зондодержателем диаметром $1.6 \mathrm{~mm}$ являются бесстолкновительными [10]. С учетом близости изучавшейся плазмы к максвелловской среде в условиях данного эксперимента можно считать примерно справедливыми упомянутые выше формулы Бома (1) и Больцмана (2), а также „закон 3/2““ в форме уравнения CLB (5).

\section{Результаты специального эксперимента и их анализ}

С помощью формулы Больцмана (2) были найдены радиальные распределения плотности ионного тока $j_{i f}(r)$ 


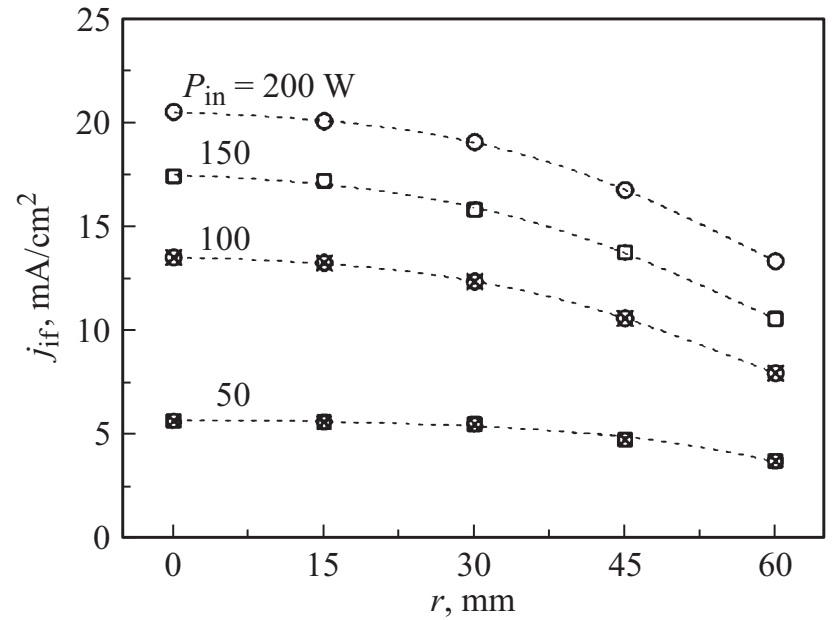

Рис. 4. Радиальные распределения плотности ионного тока на цилиндрический зонд под плавающим потенциалом.

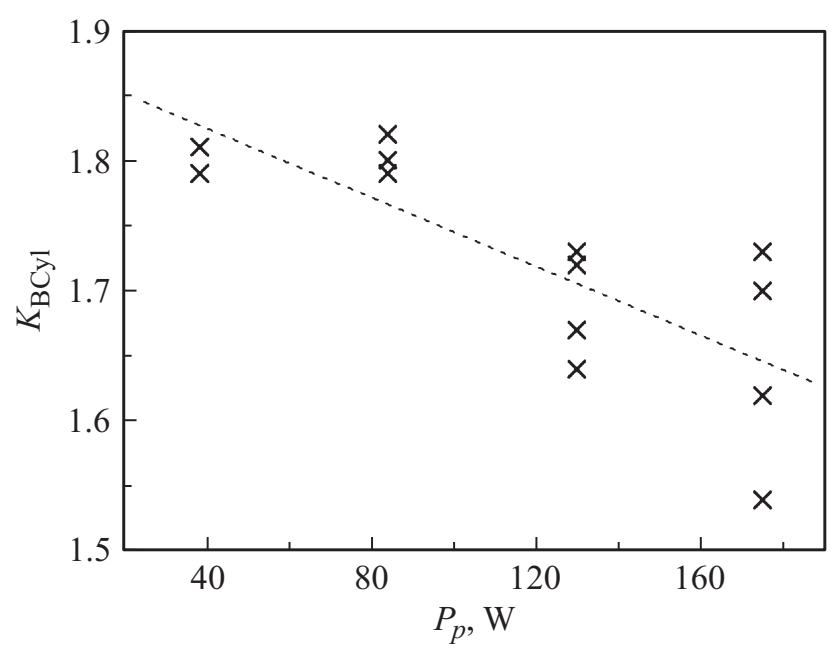

Рис. 5. Зависимость $K_{\mathrm{BCyl}}$ от ВЧ мощности $P_{p}$, поглощенной плазмой разряда.

на зонд под плавающим потенциалом с использованием измеренных величин плотностей тока электронного насыщения $j_{e s}$, электронных температур $T_{e}$ и разностей зондовых потенциалов $\Delta V_{f}$, полученных из приведенных в [10] данных по $V_{s}(r)$ путем вычитания из $V_{s}$ плавающего потенциала цилиндрического зонда $V_{f}=4.39 \mathrm{~V}$, который оказался постоянным на всех режимах разряда. Результаты по $j_{i f}(r)$ для $P_{\text {in }}=50-200 \mathrm{~W}$ показаны на рис. 4.

С помощью формулы (4) на основании этих данных и измеренных $n_{e}$ и $T_{e}([6]$ и [10]) были найдены значения составных коэффициентов Бома, которые представлены на рис. 5 для различных величин поглощенной плазмой ВЧ мощности $P_{p}$, найденных в [10] по результатам интегральной диагностики изучаемого устройства.

Видно, что в целом она имеет падающий характер, на что указывает линейная аппроксимация зависимости $K_{\mathrm{BCyl}}\left(P_{p}\right)$.
Вначале толщины зондовых слоев оценивались в приближении CLB-модели зондового слоя. Согласно уравнению (5), выражение для произведения $x_{\mathrm{CBL}} A_{\mathrm{L}}$ записывается следующим образом:

$$
\begin{aligned}
x_{\mathrm{CLB}} A_{\mathrm{L}} & =\left(4 \varepsilon_{0} / 9\right) \Delta V_{f}^{3 / 2} / K_{\mathrm{BCyl}} a^{2} e n_{e} T_{e}^{1 / 2} \\
& =2.4564 \cdot 10^{7} \Delta V_{f}^{3 / 2} / K_{\mathrm{BCyl}} a^{2} n_{e} T_{e}^{1 / 2} .
\end{aligned}
$$

Левая часть этого выражения представляет собой функцию от $x_{\mathrm{CLB}}$, которая была определена табличными данными [5] и аппроксимирована в диапазоне $x_{\mathrm{CLB}}=1.1-2.0$ функцией:

$$
x_{\mathrm{CLB}} A_{\mathrm{L}}=2.0073 x_{\mathrm{CLB}}^{2}-4.3755 x_{\mathrm{CLB}}+2.3905 .
$$

Объединение уравнений (6) и (7) приводит к получению квадратного уравнения относительно $x_{\text {textCLB }}$ :

$$
\begin{aligned}
2.0073 x_{\mathrm{CLB}}^{2} & -4.3755 x_{\mathrm{CLB}}+2.3905 \\
& -2.4564 \cdot 10^{7} \Delta V_{f}^{3 / 2} / K_{\mathrm{CLB}} a^{2} n_{e} T_{e}^{1 / 2}=0 .
\end{aligned}
$$

Его решение для полного набора величин $K_{\text {ВСуl }}=$ $=1.54-1.82$ позволяет оценить толщины зондового слоя в диапазоне $x_{\mathrm{CLB}}=1.44-1.86$ при разных величинах $P_{p}$.

Однако использованная в данном расчете CLB-модель зондового слоя предполагает заполнение его пространства только ионами при полном отталкивании электронов. Это означает, что падение напряжения на зондовом слое $\Delta V_{f}$ должно существенно превышать верхнюю границу энергии высокоэнергичных электронов плазмы. Однако в условиях настоящего эксперимента верхняя граница энергии электронов находится на уровне не ниже $25 \mathrm{eV}$, что видно из рис. 3, тогда как напряжение на зондовом слое при плавающем потенциале зонда $\Delta V_{f}=V_{s}-V_{f}$ не превышает $17 \mathrm{~V}$ [10]. Следовательно, некоторое количество высокоэнергетических электронов могло проникать в зондовый слой, изменяя его параметры.

С подобной ситуацией столкнулись авторы работы [11], в которой параметры аргоновой плазмы изучались с помощью $U$-образного зонда, для которого весьма важно знание реальных толщин зондового слоя. Сначала они были оценены по CLB-модели зондового слоя, а затем корректировались на основании так называемой „ступенчато-фронтальной“ (SF) модели, допускающей проникновение в зондовый слой некоторого количества высокоэнергетических электронов плазмы. Обработка данных работы [11] позволила получить зависимость $x_{\mathrm{SF}}=f\left(x_{\mathrm{CLB}}\right)$, представленную на рис. 6 .

Эти данные в представленном диапазоне $x_{\mathrm{CLB}}$ с высокой точностью аппроксимировались прямой линией

$$
x_{\mathrm{SF}}=0.692 x_{\mathrm{CLB}}+0.3816 \text {, }
$$

характеризующей некоторое снижение $x_{\mathrm{SF}}$ по сравнению со значениями $x_{\text {CLB }}$. Результаты такой корректировки 


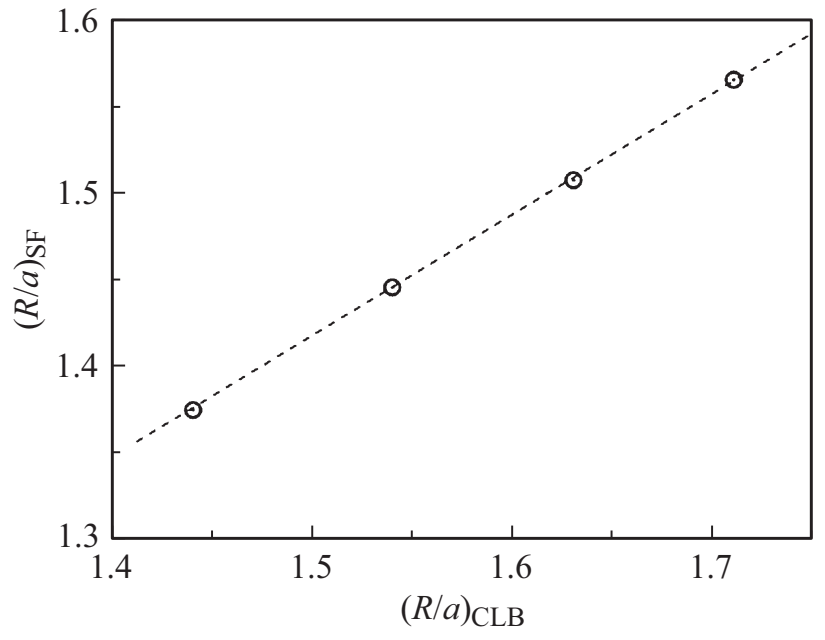

Рис. 6. Зависимость $x_{\mathrm{SF}}$ от $x_{\mathrm{CLB}}$ по данным [11].

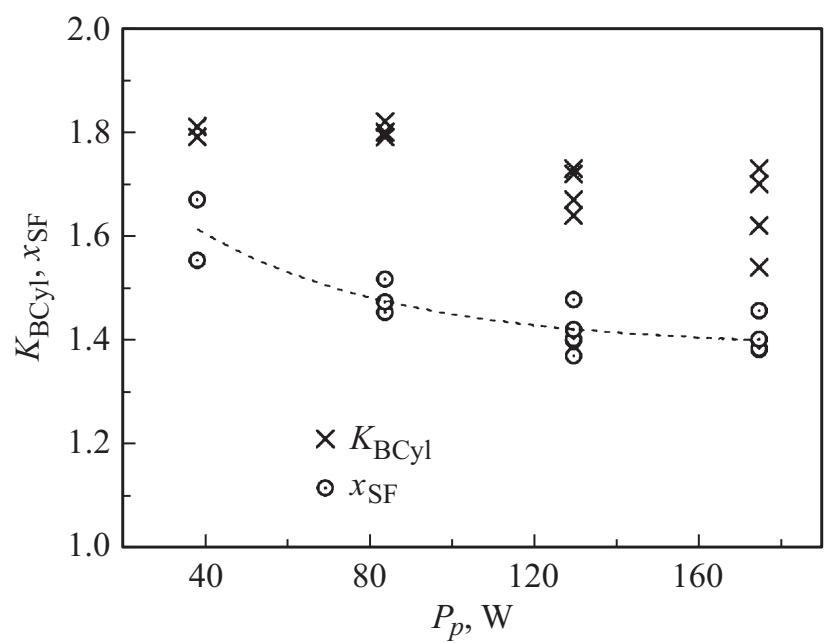

Рис. 7. Зависимость скорректированных значений $x_{\mathrm{SF}}$ и составных коэффициентов Бома $K_{\mathrm{BCyl}}$ от $P_{p}$.

экспериментальных данных настоящей работы по $x_{\mathrm{CLB}}$ представлены на рис. 7 в виде кружков.

Их аппроксимация штриховой линией подтверждает снижающийся характер зависимости $K_{\mathrm{BCyl}}\left(P_{P}\right)$ при некотором уточнении ее формы, поскольку график $x_{\mathrm{SF}}\left(P_{P}\right)$ оказался более четким по сравнению с перенесенными сюда из рис. 5 данными по составному коэффициенту Бома $K_{\mathrm{BCyl}}$.

Далее задача определения $C_{\mathrm{BCyl}}$ могла быть решена двумя путями. Графический путь состоял в подборе коэффициента $C_{\mathrm{BCyl}}>1$ к аппроксимирующей функции $x_{\mathrm{SF}}\left(P_{P}\right)$ на рис. 7 с целью смещения ее вверх до усреднения всей группы данных по $K_{\mathrm{BCyl}}$. Этот путь был использован в работе [12] и привел к получению $C_{\mathrm{BCyl}} \approx 1.23$. Второй арифметический путь состоит в вычислении значений коэффициента Бома $C_{\mathrm{BCyl}}=K_{\mathrm{BCyl}} / x_{\mathrm{SF}}$ для всех экспериментальных точек $K_{\mathrm{BCyl}}$, представленных на рис. 8.
Здесь нанесена сплошная прямая линия $C_{\mathrm{BCyl}}=1.23$ с обозначением поля разброса $\pm 7 \%$. Видно, что арифметический путь получения уточненного коэффициента Бома подтвердил графический результат работы [12].

Теперь можно окончательно сформулировать предложенную в настоящей работе методику зондовых измерений толщин зондовых слоев и средней массы ионов с включением экспериментально обоснованных практических формул:

1) Контроль вида EEDF плазмы.

2) Уточненный коэффициент Бома $C_{\mathrm{BCyl}}=1.23$ видоизменяет формулу (6):

$$
X_{\mathrm{CLB}}^{2} A_{\mathrm{L}} \approx 1.997 \cdot 10^{7} \Delta V_{f}^{3 / 2} / a^{2} n_{e} T_{e}^{1 / 2} .
$$

Табличные данные [5] относительно $A_{\mathrm{L}}$ определяют зависимость $x_{\mathrm{CLB}}^{2} A_{\mathrm{L}}=f\left(x_{\mathrm{CLB}}\right)$, которая для $x_{\mathrm{CLB}}=1.1-2.0$ с хорошей точностью была аппроксимирована полиномом 4-й степени

$$
\begin{aligned}
X_{\mathrm{CLB}}^{2} A_{\mathrm{L}} & =0.4756 x_{\mathrm{CLB}}^{4}+0.05815 x_{\mathrm{CLB}}^{3}+(-2.093) x_{\mathrm{CLB}}^{2} \\
& +2.1202 x_{\mathrm{CLB}}-0.56165 .
\end{aligned}
$$

Объединяя выражения (10) и (11), получаем уравнение для оценки $x_{\mathrm{CLB}}$ :

$$
\begin{aligned}
0.4756 x_{\mathrm{CLB}}^{4} & +0.05815 x_{\mathrm{CLB}}^{3}-2.093 x_{\mathrm{CLB}}^{2}+2.1202 x_{\mathrm{CLB}} \\
& -0.56165-1.997 \cdot 10^{7} \Delta V_{f}^{3 / 2} / a^{2} n_{e} T_{e}^{1 / 2}=0 .
\end{aligned}
$$

Оно без особых трудностей решается, например, с помощью доступных в Интернете „калькуляторов онлайн“.

3) Полученные величины $x_{\text {CLB }}$ корректируются формулой (9) для приведения их в соответствие с SF-моделью зондового слоя объемного заряда, определяющей толщины этого слоя $\delta=R_{\mathrm{SF}}-a=a\left(x_{\mathrm{SF}}-1\right)$. Их знание важно в плане контроля корректности зондовой теории, используемой для интерпретации зондовых BAX.

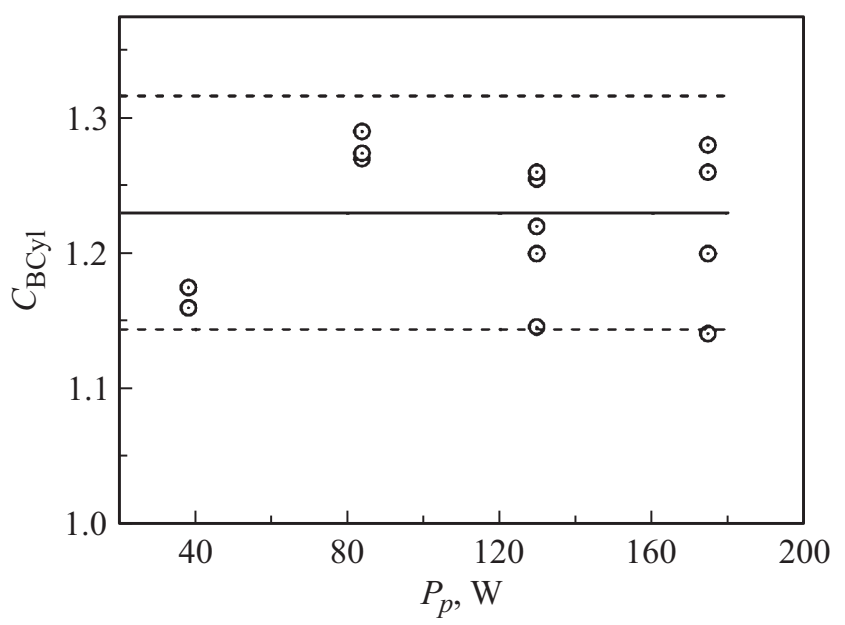

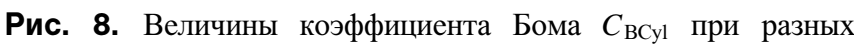
значениях $P_{p}$. 
4) Коэффициент Бома $C_{\mathrm{BCyl}}=1.23$ видоизменяет также формулу (4), определяющую выражение для нахождения массы ионов:

$$
M_{i}=3.026 x_{\mathrm{CLB}}^{2} e^{3} n_{e}^{2} T_{e} / j_{i f}^{2} .
$$

В случае работы с газовой смесью это будет средняя ионная масса, а при использовании определенного газа этот результат определит степень его чистоты.

\section{Выводы}

1. Предложено расширение зондовой диагностики максвелловской плазмы измерением коэффициента Бома, толщины зондового слоя и массы ионов плазмы.

2. Аккуратная зондовая диагностика ксеноновой плазмы ВЧИ разряда при давлении 2 mTorr с помощью цилиндрического зонда и системы Plasma Sensors VGPS-12 позволила определить коэффициент Бома $C_{\mathrm{BCyl}}=1.23$ в классической формуле Бома, что послужило основой для практической формулировки предложенной методики.

Авторы считают своим приятным долгом выразить признательность проф. В.А. Годяку и его коллеге В.М. Александровичу за поддержку данного исследования и оказание активной помощи в проведении экспериментов.

\section{Список литературы}

[1] Godyak V.A., Demidov V.I. // J. Phys. D: Appl. Phys. 2011. Vol. 44. P. 233001.

[2] Электронный ресурс. Режим доступа: // VGPS Probe System. URL: http://www.plasmasensors.com

[3] Riaby V.A., Obukhov V.A., Masherov P.E. // High Voltage Engineering. 2012. Vol. 38. Suppl. P. 790-793.

[4] Bohm D. Characteristics of Electrical Discharges in Magnetic Fields / Ed. by A. Guthrie, R.K. Wakerling. NY.-TorontoLondon: McGraw-Hill Book Co., Inc., 1949. P. 1-49.

[5] Козлов О.В. Электрический зонд в плазме. М.: Атомиздат, 1969. C. $20-21$

[6] Masherov P.E., Riaby V.A., Godyak V.A. // Rev. Sci. Instrum. 2016. Vol. 87. P. 2 B926.

[7] Godyak V. Inductive plasma source with high coupling efficiency. US Patent 8444870. Int. Cl. H01J 37/3211, C23F 1/00, 21 May 2013.

[8] Godyak V.A. // Plasma Sources Sci. T. 2011. Vol. 20. P. 025004.

[9] Машеров П.Е. // Вестник Московского авиационного института. 2016. Т. 23. № 2. С. 42-49.

[10] Рябый В.А., Машеров П.Е. // Изв. РАН. Энергетика. 2016. № 2. C. $45-57$.

[11] Piejak R.B., Godyak V.A., Garner R., Alexandrovich B.M. // J. Appl. Phys. 2004. Vol. 95. N 7. P. 3785-3791.

[12] Masherov P.E., Riaby V.A. // Rev. Sci. Instrum. 2016. Vol. 87. P. 086106. 